\title{
Multilingual practices and language scaling in behavioural change communication on HIV/AIDS in Lesotho: The case of Phela health and development communications
}

\author{
Konosoang Sobane \\ Department of General Linguistics, Stellenbosch University, Private Bag X1, 7602 Matieland, South Africa \\ E-mail: msobane@gmail.com
}

\begin{abstract}
This research report provides an account of how multilingualism is handled in behavioural change communication about HIV/AIDS by Phela health and development communications, an NGO in Lesotho. The report discusses the distribution of languages in the print and media publications of the NGO. It highlights the bilingual nature of these publications, which emphasise English and Sesotho as enshrined in the constitution, and which exclude other minority languages of the country. The report discusses the implications on this approach to language on minority language speakers, more especially in the context of HIV/AIDS, a pandemic which affects all the population regardless of language. The report lastly makes recommendations for a more inclusive approach in behavioural change communication about HIV/AIDS.
\end{abstract}

Keywords: multilingualism, behaviours, communication

\section{Introduction}

In cognisance of the escalating statistics of HIV/AIDS prevalence and the negative impact that it has on human and economic resources in Lesotho, the government and non-governmental organisations (NGOs) have had to respond proactively by introducing several interventions that can reduce the infection rate, with particular emphasis on the distribution of behavioural change communication (BCC). This type of communication is intended to influence the social and sexual behaviours of the target population and ultimately reduce the spread of HIV/AIDS. The role of BCC is explained further by Bessinger, Katende and Gupta (2004:398-99), who note that BCC activities are aimed at promoting the use and acceptance of condoms for disease prevention, as well as educating the population about matters such as prevention of HIV infection.

The successes of BCC in reducing HIV infection rates have been reported in countries outside Africa such as Thailand (see Nelson, Celentano, Eiumtrakol, Hoover, Beyrer, Suprasert, Kuntolbutra and Khamboonruang 1996) as well as in Africa (see Katende, Bessinger, Gupta, Knight and Lettenmaier 2000; Vaughn, Rogers, Singhal and Swahele 2000). These successes have been attributed to the fact that BCC employs the use of multimedia which is expected to reach a larger population than if the health messages were distributed by health personnel in health centres (Africa, Buseh, Glass, Mcelmurry, Mkhabela and Sukati 2002:397). The use of broadcast media 
also ensures that the information reaches the youth and adolescents who are reported as the most vulnerable group in most countries (cf. Ansell 2009, Bass 2006, Sukati, Mndebele and Hons 2005).

Although there is ample literature on the types of media most suitable for BCC messages, the success rates of BCC in different countries, and the accessibility of such campaigns, researchers have neglected evaluating the multilingual practices that are employed in conveying these messages. This area is important because most countries in which these messages and campaigns are carried out are multilingual and HIV/AIDS affects speakers of all languages. Moreover, the way an organisation distributes languages provides insight into how multilingualism is construed and linguistic diversity is managed in that organisation and the multilingual spaces around it (Blommaert, Collins and Slembrouck 2005). In particular, there seems to be no literature evaluating how language diversity is incorporated in order to make the messages linguistically available to speakers of different languages, particularly in Lesotho where the prevalence of HIV/AIDS is very high. This research note is aimed at bridging this knowledge gap by reporting on the multilingual practices of Phela Health and Development Communications (PHDC), an NGO which distributes BCC information in Lesotho.

PHDC is an organisation that was established in Lesotho in 2002. According to their evaluation document entitled "Evaluating PHDC 2002-2007", this NGO is a behaviour change organisation, which mainly develops and distributes behaviour change communication materials on HIV/AIDS with the aim of influencing behaviour change and ultimately combating the spread of the disease. The basic way in which PHDC works is to develop and distribute information through different modes of communication such as literary materials, radio and television dramas. In all of these materials, PHDC advocates for social and behaviour changes that lead to healthy lifestyles and prevention of HIV/AIDS. The authors of "Evaluating PHDC 2002-2007" further report that the target population of the organisation is the general population of Lesotho aged between 18 and 45 . This implies that the materials are meant for this population regardless of what language they speak.

Even though this report provides a detailed account of how these resources were distributed as well as some of PHDC's successes and failures, there is no insight into either the distribution of linguistic resources in these different modes, or into how linguistic diversity is accommodated in conveying BCC messages. The present research is aimed at reporting on the multilingual practices of PHDC by examining how the different materials used by the NGO distribute linguistic resources, and whether the materials cater for language diversity in a country which is seemingly a multilingual space.

\section{An overview of Lesotho's multilingual landscape}

Lesotho does not have a written language policy except a clause in the constitution which recognises Sesotho and English as official languages. According to Khati (1996:2), Sesotho is the first language of approximately 98 percent of the population and is used widely as a medium of communication in day-to-day interactions and in matters such as politics, religion, and the mass media. English is spoken by approximately 30 percent of the population and it is reserved for official interactions such in the legislative, administrative and the educational sectors, in which it is a medium of instruction in schools from primary level. Most speakers of English acquired the language through formal education and therefore it is a second language to them. 
Several researchers working on Lesotho's language situation (see Lynn 1994, Khati 1996, Matsoso 2000, Moloi and Matsau 2011) have observed that, apart from Sesotho and English, a considerable fraction of the population living in the northern and eastern parts of the country, across the borders of South Africa speak Nguni languages such as isiXhosa, isiZulu, Ndebele and Sephuthi as their mother tongue. Moloi and Matsau (2011:68) note that isiXhosa is the most widely spoken of all of these languages. There are also a few Asian and Indian language speakers in the country. Although some speakers of these minority and migrant languages also speak Sesotho and English, there are some who are monolingual.

On the basis of this linguistic landscape, Lesotho is multilingual like most African countries. Despite the diverse linguistic repertoires of the country's population, citizens have the same healthrelated challenges and are equally affected by HIV/AIDS. Since they have a stake in the economy of the country, interventions to reduce the infection rates of this pandemic by NGOs such as PHDC are also expected to benefit them. However PHDC's policy on the target population does not have a specific language clause so their multilingual practices are still not known.

\section{Multilingualism in the health sector}

Research on multilingualism (Blommaert et al. 2005, House and Rehbein, 2004, Collins and Slembrouck 2006), acknowledges multilingualism as a growing trend, emerging from increased mobility and migration processes in different societies. Moyer (2010:2) defines multilingualism as "the presence of two or more languages with different social statuses in a given speech community". Multilingualism demands that communication practices be geared towards accommodating the diverse linguistic repertoires, especially in the health sector. Since health institutions are the ones responsible for the scaling (or distribution) of languages, (Collins and Slembrouck 2006), they are the ones responsible for the success of multilingual health communication.

At the forefront of guaranteeing the success of multilingual health communication is acknowledgement and use of minority languages in health organisations and institutions (cf. Bischoff, Bovier, Rrusteni, Garriazano, Eytan and Loutan 2003, Cioffi 2003, Harmsen, Meeuwesen, Van Wieringen, Bernsen and Bruijnzeels 2003, Bernard, Whitaker, Ray, Rockich, Barton-Baxter, and Barnes 2006, Deumert 2010). Although most research on multilingual health communication focuses on doctor-patient interactions, the findings can be generalised to all forms of healthcare and health education communication, including BCC. There is a consensus among the aforementioned authors that when healthcare is carried out in a patient's foreign language, positive health outcomes are indeed compromised. Some of the problems that occur when healthcare is not carried out in one's own language include the silencing of patients' voices thereby rendering them unable to explain their problems (Deumert 2010:58); misunderstanding of the health condition (Harmsen et al. 2003:108); and general noncompliance to treatment as a result of a lack of understanding (Bischoff et al. 2003:508). These findings suggest that if healthcare and education is to become accessible and effective, the problems that language barriers pose will have to be addressed.

As a way of addressing language barriers, many health institutions use different forms of interpreting services. Dressler and Pils (2009) identified the use of family members, cleaners and even kitchen staff as interpreters. In a study carried out in South Africa, Drennan and Swartz (2002) discovered that certain hospitals used an array of bilingual hospital staff such as nursing staff, ward cleaners and security personnel, while Angelelli (2004) established that professional 
interpreters are used. Apart from interpreting, other healthcare institutions use literary materials such as bilingual translation manuals, health dictionaries and bilingual word-phrase lists (cf. Collins and Slembrouck 2006, Moyer 2010).

Although all of these methods have their respective disadvantages, there is consensus among researchers that the language barrier impedes quality healthcare and effective health education, and that healthcare and health education is more effective when language diversity is properly managed. Since BCC is concerned largely with health education, it could be expected that when it is carried out in a multilingual setting, it faces the same risks of being misunderstood and therefore it has to be carried out with awareness of the linguistic diversity of the population at which it is targeted.

The challenges of BCC become compounded in matters relating to HIV/AIDS because of the nature of this pandemic. Research on HIV/AIDS acknowledges the fact that although the best way to combat the spread of the disease is through communication about prevention and care, this kind of communication is still marred by dilemmas and challenges (cf. Curtis and Patrick 1997, Aujoulat, Linion, Bois, Martin and Deccache 2002, Ijadunola 2007). These challenges include the fact that HIV communication entails topics that are difficult to broach, particularly in the African culture. Examples of such topics are sexuality (cf. Helleve et al. 2009:197), the prospect of death (cf. Aujoulat 2002:217, Ijadunola 2007:80), and the stigmatisations which are still associated with the disease (cf. Curtis and Patrick 1997, Kohi, Makoae, Chirwa, Holzemer, Phetlu, Uys, Naidoo, Dlamini, and Greef 2006, and Ijadunola 2007).

It is clear from the discussion regarding the literature above that although $\mathrm{BCC}$ is an effective tool in curbing the spread of HIV/AIDS, its application in multilingual settings has to be done with consciousness of the linguistic diversity of the target population. While this literature lays an important foundation regarding issues of access to healthcare and health education for people who do not speak majority languages in different countries, it does not say anything about access to $\mathrm{BCC}$ in the mother tongue.

\section{Methodology}

The research design is a case study investigating the multilingual practices of one NGO in order to relate it to other NGOs which do the same work. Data was collected from PHDC officials in Maseru and from first language speakers of Ndebele, isiXhosa and isiZulu who also speak either Sesotho or English as a second language, and who originate from the rural areas of Lesotho. Purposive selection of the participants that was based mostly on availability resulted in a sample of two PHDC officials, and two speakers of each of the aforementioned languages, originating from the districts of Mokhotlong, Quthing and Qachas Nek respectively. The method of data collection was semi-structured interviews coupled with collection of PHDC print publications and evaluation reports. The interviews were analysed in terms of content for themes relating to the research question, after which document analysis of the collected literary materials was carried out.

\section{Findings and discussion: Language scaling in the PHDC materials}

PHDC has produced a combination of print, radio and television materials that are targeted at Lesotho citizens above 18 years of age. According to the "Impact Evaluation Summary of the Soul City Institute Regional Programme 2002-2007”, PHDC has produced three booklets 
namely "HIV/AIDS Action Now", "Mother and Child Care", and "Help Stop Violence Against Women". The organisation distributed between 670,000 and 890,000 of each of these booklets. The booklets contain educational messages on HIV prevention, care and support of those infected, motherhood and breastfeeding for HIV positive women, and women's rights and abuse. All of these booklets have both Sesotho and English versions.

Information in these booklets is presented in two styles. The first has a conversational style with pictures of the interlocutors. The conversations are followed by detailed explanations of key information. Figure 1 is an example of an extract from one of the booklets.

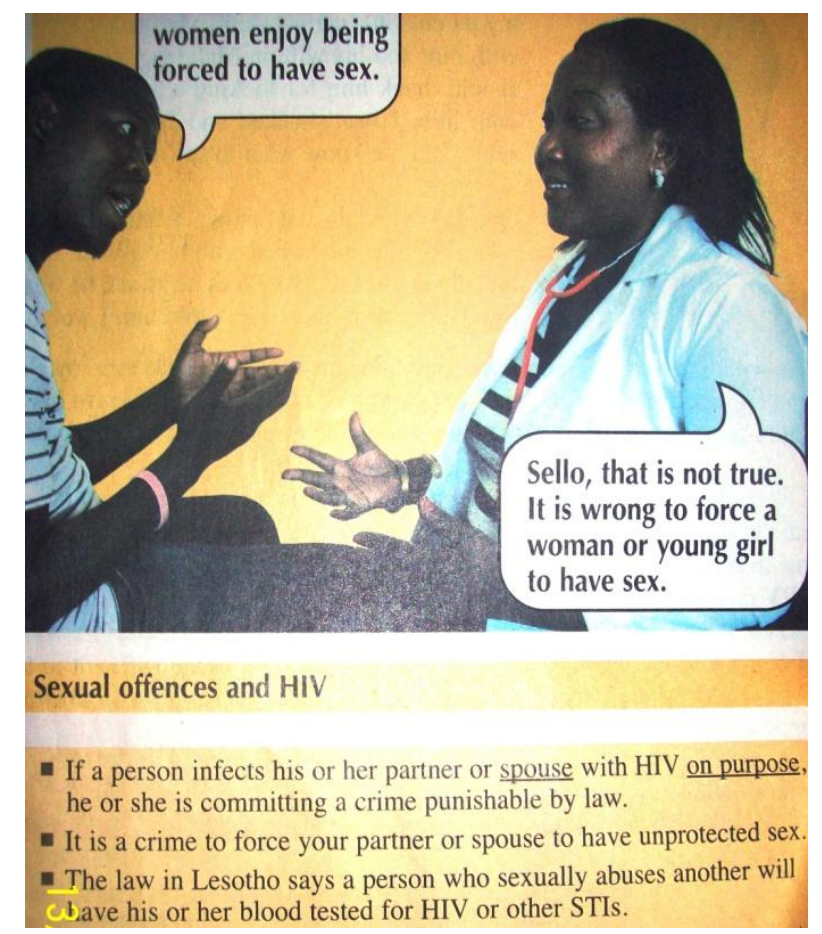

Figure 1. Extract showing the conversation and the explanation of key points.

In the English booklets, some key words, which may be unfamiliar to the reader, have been underlined and translated into Sesotho in the margins of the text. These translations are meant to make it easy for a bilingual speaker of English and Sesotho, who is not highly proficient in English, to understand the text. The Sesotho version of the booklet does not contain these translations.

The second style is a narrative style in which a real life story is told. This is immediately followed by advice or a summary of the story. The advice and the summary are given in the language of the text, that is, English for English booklets and Sesotho for Sesotho booklets. In both of the conversations and the stories, there is no provision for other languages apart from the two aforementioned languages, neither in terms of translation of key words nor in the summaries and advices.

\subsection{Radio dramas}

The "Impact Evaluation Summary of the Soul City Institute Regional Programme 2002-2007" reports that PHDC has produced two radio drama series. The first one was titled "Musa Pelo" (loosely translated as "The Heart Comforter"). It has 60 episodes that contain BCC messages on mother and childcare, HIV/AIDS, PMTCT and child abuse. This drama was aired between 
November 2005 and March 2006. The second drama series is "Mafube a Bophelo" (loosely translated as "Rays of Life") which comprised of 45 episodes of 15 minutes each and was broadcast between March and June of 2007. Amongst other topics, this drama included messages on HIV and AIDS treatment and support, smoking and youth empowerment. Both radio dramas were broadcast exclusively in Sesotho on Radio Lesotho, a state-owned radio station that has a very wide coverage.

\subsection{Television documentaries}

Information from the PHDC evaluation report shows that PHDC produced two local documentary series consisting of 13 episodes of 15 minutes each. These were broadcast nationally in 2004 and 2005. These television documentaries contained BCC messages on issues such as HIV and AIDS, sexuality, breastfeeding, prevention, care and support, and community development issues. The documentaries were in Sesotho and some of them had English subtitles.

\section{Multilingual practices to acknowledge linguistic diversity}

A close look at language practices reflected in PHDC BCC shows that the organisation operates within the perimeters of the bilingual policy enshrined in the constitution of Lesotho which recognises Sesotho and English as co-official languages. Although the publications do not make any provision for linguistic diversity that extends beyond this multilingualism, because of scarcity of resources, it has been established that the NGO has strategies that they believe can help accommodate speakers of minority languages.

The first strategy is the use of pictures in the booklets along with the stories and messages. These pictures are expected to convey the message even when the reader does not understand the language. A PHDC official explains the inclusion of pictures in the booklets by saying:

...they are made to make the resources more interesting to read ... but even when you cannot read you can still follow and I guess even speakers of those other minority languages can get the message that way.

This implies that the primary purpose of the pictures was to aid illiterate people, whereas accommodating linguistic diversity seems to be secondary.

In examining the pictures in the booklets, one does not see any messages embedded in most of the pictures. For example, the picture in Figure 1 does not convey anything about forced sex and how wrong it is. Therefore, if one cannot read the text, the picture will not act as a secondary source to convey the message. These pictures are therefore not instrumental in conveying messages to speakers of other languages other than Sesotho and English.

Another way in which PHDC expects speakers of other languages to access the BCC messages with their available resources is through translation. Since most people who have been through the school system know Sesotho and English, it is expected that the youth will translate for the elder population who are not proficient in these languages. This is regardless of the fact that the youth may not be proficient enough to carry out this translation. Translation of the booklets has been found to pose challenges due to being intergenerational and because of the nature of the information contained in these booklets, which include themes such as sexual conduct or behaviour. The following is an excerpt from one of the booklets: 
A: Mpho, Tau refuses to test and yet he wants to have unprotected sex.
B: $\quad$ Malika it is not safe to have unprotected sex...

While this is a valuable and educational message, it would be very difficult or uncomfortable for a child to translate it to his or her parents. In addition, the page adjacent the one containing this message, includes illustrations on how to use a condom. This content makes it even more difficult for these two generations to read the book together because, culturally, the older generation does not discuss such issues with the younger generation in a family setting. This situation still leaves minority language speakers unable to access the BCC messages conveyed by PHDC.

The radio dramas are strategically aired after school hours when schoolchildren are available to translate for their elders (however, it cannot be guaranteed that this population is able to access the information). In families where the child is available, it has been established that it is difficult for him or her to conduct simultaneous interpreting, so a summary of the episode is provided at the end. The question remains, then, as to how the child decides which pieces of information to omit, given their proficiency levels in both Sesotho and English. Furthermore, one also has to wonder what happens to those individuals who do not have children to interpret these stories for them.

\section{Conclusion}

These findings show that PHDC messages are not easily accessible to speakers of languages other than Sesotho and English. Although its evaluation reports high rates of success in terms of improving sexual behaviour, these very valuable messages are not made to reach a considerable fraction of the population, thus making it impossible to benefit from them. The options of pictures and translations which are expected to help minority language speakers all have different challenges. While this research has focused its attention on PHDC, the linguistic practices in conveying BCC are characteristic of all NGOs and government sectors who work with HIV/AIDS and related issues in the country. There is much emphasis on the use of English and Sesotho in HIV/AIDS education, with total disregard of the multilingual nature of Sesotho. This emphasis of bilingualism is contrary to the multilingual situation of Lesotho.

These findings also lay a foundation for a more inclusive approach to BCC. Instead of producing a large number of booklets in just two languages, it is recommended that more booklets be produced in other languages and distributed in places where these speakers are found, for example, distribute isiXhosa booklets in Quthing. It would also be ideal to consult with speakers of these languages in order to establish viable solutions to ensure that they may benefit from the widespread HIV/AIDS education in Lesotho.

\section{References}

Africa, S., A.G. Buseh, L.K. Glass, B.J. Mcelmurry, M. Mkhabela and N.A. Sukati. 2002. Primary and preferred sources for HIV/AIDS and sexual risk behaviour information among adolescents in Swaziland. International Journal of Nursing Studies 39: 525-538.

Aujoulat, I., F. Libion, H. Bois, F. Martin and A. Deccache. 2002. Communication difficulties of healthcare professionals and family caregivers of HIV infected patients. Patient Education and Counselling 47: 213-222. 
Angelelli, C.A. 2004. Medical interpreting and cross cultural communication. Cambridge: Cambridge University Press.

Ansell, N. 2009. Producing interventions for AIDS-affected young people in Lesotho's schools: Scalar relations and power differentials. Geoforum 40(4): 675-685.

Bass, E. 2006. WHO and UNAIDS set controversial course for HIV testing. The Lancet Infectious Diseases 6(12): 760-766.

Bernard, A., M. Whitaker, M. Ray, A. Rockich, M. Barton-Baxter and S.L. Barnes. 2006. Impact of language barrier on acute care medical professionals is dependent upon role. Journal of Professional Nursing: Official Journal of the American Association of Colleges of Nursing 22(6): 355-358.

Bessinger, R., C. Katende and N. Gupta. 2004. Multi-media campaign exposure effects on knowledge and use of condoms for STI and HIV/AIDS prevention in Uganda. Evaluation and Program Planning 27: 397-407.

Bischoff, A., P. Bovier, I. Rrustemi, F. Gariazzo, A. Eytan and L. Loutan. 2003. Language barriers between nurses and asylum seekers: their impact on symptom reporting and referral. Social Science and Medicine 57(3):503-512.

Blommaert, J., J. Collins and S. Slembrouck. 2005. Spaces of multilingualism. Language and Communication 25(3). 197-216.

Cioffi, R.N. 2003. Communicating with culturally and linguistically diverse patients in an acute care setting: Nurses' experiences. International Journal of Nursing Studies 40: 299-306.

Collins, J. 2006. Institutional procedure and literacy practice in neighbourhood health clinics in urban Flanders. Journal of Linguistic Anthropology 16(2): 249-268.

Curtis, J.R. \& D.L. Patrick. 1997. Barriers to communication about end-of-life care in AIDS patients. Journal of General Internal Medicine 12: 736-741.

Deumert, A. 2010. "It would be nice if they could give us more language": Serving South Africa's multilingual patient base. Social Science \& Medicine 71(1): 53-61.

Drennan, G. and S. Swartz. 2002. The paradoxical use of interpreting in psychiatry. Social Science \& Medicine 54(12): 1853-1866.

Dressler, D. and P. Pils. 2009. A qualitative study on cross-cultural communication in postaccident in-patient rehabilitation of migrant and ethnic minority patients in Austria. Disability and Rehabilitation 31(14): 1181-1190.

Evaluating Phela Health and Development Communication 2002-2007. [Online] Available: http://www.soulcity.org.za/research/evaluations/regionalprogramme/Evaluation $\% 20$ Report $\% 20-\% 20$ Lesotho.pdf

Harmsen, H., L. Meeuwesen, J. van Wieringen, R. Bernsen, and M. Bruijnzeels. 2003. When cultures meet in general practice: intercultural differences between GPs and parents of child patients. Patient Education and Counseling 51(2): 99-106.

Helleve, A., A.J. Flisher, H. Onya and W. Mukoma. 2009. South African teachers' reflections on the impact of culture on their teaching of sexuality and HIV/AIDS. Health 11(2): 189-204.

Ijadunola, K.T., M.Y. Ijadunola, T.C. Abiona, O.O. Odu and A.A.Akinyemi. 2007. Correlates of physicians' attitudes toward communication with HIV AND AIDS patients in Ile-Ife, Nigeria. The European Journal of Contraception and Reproductive Health Care 12(1): 76-85.

Katende, C., R. Bessinger, N. Gupta, R. Knight and C. Lettenmaier. 2000. Uganda delivery of improved services for health evaluation surveys 1999. MEASURE Evaluation Technical Report Series 6.

Khati, T. 1996. The problems and prospects of the use of African national languages in education. Unpublished manuscript. National University of Lesotho 
Lynn, T. 1994. The language situation of Lesotho today: A preliminary survey. NUL Journal of Research 4: 1-58.

Matsoso, L. 2000. Lesotho's national language policy in the silent of minority Basotho. Unpublished manuscript. National University of Lesotho

Moloi, F.L and A.M. Matsau. 2011. Lesotho language policy and its impact on minority languages speakers. Proceedings of the $10^{\text {th }}$ LASU Conference 2009. Roma, Lesotho.

Moyer, M.G. 2010. What multilingualism? Agency and unintended consequences of multilingual practices in a Barcelona health clinic. Journal of Pragmatics 43(5): 12091221.

Nelson, K.E., D.D. Celentano, S. Eiumtrakol, D.R. Hoover, C. Beyrer, S. Suprasert, S. Kuntolbutra and C. Khamboonruang. 1996. Changes in sexual behaviour and a decline in HIV infection among young men in Thailand. New England Journal of Medicine 335(5): 297-303.

Soul City Regional Programme Impact Evaluation Summary 2002-2007. [Online] Available: http://www.soulcity.org.za/research/evaluations/regional-programme/soul-cityinstitute-regional-programme-2002-2007-impact-evaluation-summary

Sukati, N.A., SC Mndebele and B. Hons. 2005. HIV/AIDS symptom management in Southern Africa. Journal of Pain and Symptom Management 29(2): 185-192.

Vaughn, P.W., E.M. Rogers, A. Singhal and R.M. Swalehe. 2000. Entertainment-education and HIV/AIDS prevention: A field experiment in Tanzania. Journal of Health Communication 5(Supplement 1): 81-100. 